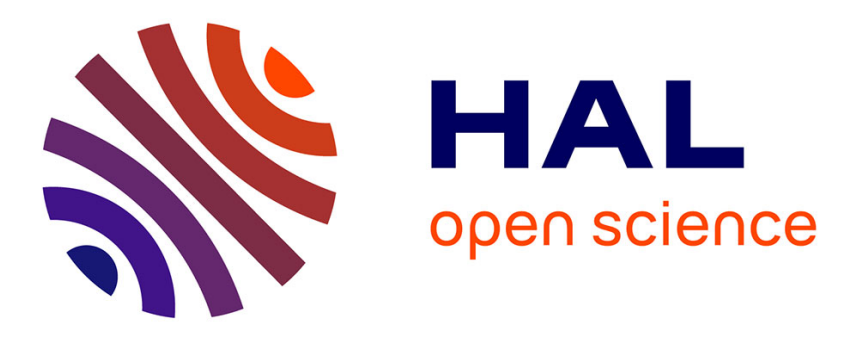

\title{
Archéologie préhispanique du littoral Pacifique nord-équatorial
}

\author{
Jean-François Bouchard
}

\section{To cite this version:}

Jean-François Bouchard. Archéologie préhispanique du littoral Pacifique nord-équatorial. Bulletin de la Société préhistorique française, 1989, 10/12, pp.393-396. 10.3406/bspf.1989.9898 . hal-02910889

\section{HAL Id: hal-02910889 \\ https://hal.science/hal-02910889}

Submitted on 8 Sep 2020

HAL is a multi-disciplinary open access archive for the deposit and dissemination of scientific research documents, whether they are published or not. The documents may come from teaching and research institutions in France or abroad, or from public or private research centers.
L'archive ouverte pluridisciplinaire HAL, est destinée au dépôt et à la diffusion de documents scientifiques de niveau recherche, publiés ou non, émanant des établissements d'enseignement et de recherche français ou étrangers, des laboratoires publics ou privés. 


\section{Archéologie préhispanique du littoral pacifique nord-équatorial} Jean-François Bouchard

\section{Citer ce document / Cite this document :}

Bouchard Jean-François. Archéologie préhispanique du littoral pacifique nord-équatorial. In: Bulletin de la Société préhistorique française, tome 86, n¹0-12, 1989. Du terrain au laboratoire : Pour un meilleur dialogue en archéologie. pp. 393396;

doi : https://doi.org/10.3406/bspf.1989.9898

https://www.persee.fr/doc/bspf_0249-7638_1989_hos_86_10_9898

Fichier pdf généré le 10/01/2019 


\title{
Archéologie préhispanique du littoral Pacifique nord-équatorial \\ 1976-1981 : Projet Tumaco (Côte sud de la Colombie) 1982-1987 : Projet La Tolita (Côte nord de l'Équateur)
}

\author{
par Jean-François Bouchard
}

Le $1^{\text {er }}$ projet (Tumaco) (fig. 1 et 2), a été effectué sur la demande de l'Institut Colombien d'Anthropologie, dans le cadre de son plan national de recherches archéologiques. Avant cette recherche, on ne disposait que de données très fragmentaires concernant cette région, étudiée par deux programmes précédents (en 1948 et 1960). Les recherches ont d'abord comporté la fouille d'un site d'habitat (Inguapi), où une séquence chrono-culturelle a été obtenue. Dans ce site, sont apparues les premières évidences de contacts étroits avec la côte du nord de l'Équateur, à moins de $100 \mathrm{~km}$ au sud de Tumaco. On a aussi pu découvrir, pour la période la plus ancienne (vers 300 av. J.-C.), les plus anciens vestiges d'orfèvrerie datés par le $14 \mathrm{C}$ pour l'ensemble de la Colombie. La fouille de quatre autres sites, depuis le rivage du Pacifique jusqu'à environ $50 \mathrm{~km}$ à l'intérieur des terres, a permis de compléter la séquence chrono-culturelle de base.

Le $2^{\mathrm{e}}$ projet (La Tolita) (fig. 1), a été réalisé sur la demande du Musée Archéologique de la Banque Centrale de l'Équateur, avec une de ses équipes de recherche. Le site étudié (Cancha $\mathrm{H}$. 9, La Tolita), et ceux découverts au cours des prospections sont caractérisés par des modèles d'établissement en bordure de cours d'eau, comme pour les sites de la région de Tumaco.

Ces deux projets successifs ont mis en évidence l'unité ayant existé entre les deux régions à divers moments de la période préhispanique. Les deux régions, voisines, correspondent à un même milieu naturel (forêt de palétuviers sur la côte et dans les estuaires, puis forêt tropicale humide dans l'intérieur de la plaine alluviale jusqu'aux collines du piémont de la cordillère occidentale). L'ensemble est compris entre le $1^{\circ}$ et le $2^{\circ}$ de latitude Nord et entre le 79'30' et le $78^{\circ} 30^{\prime}$ de longitude.

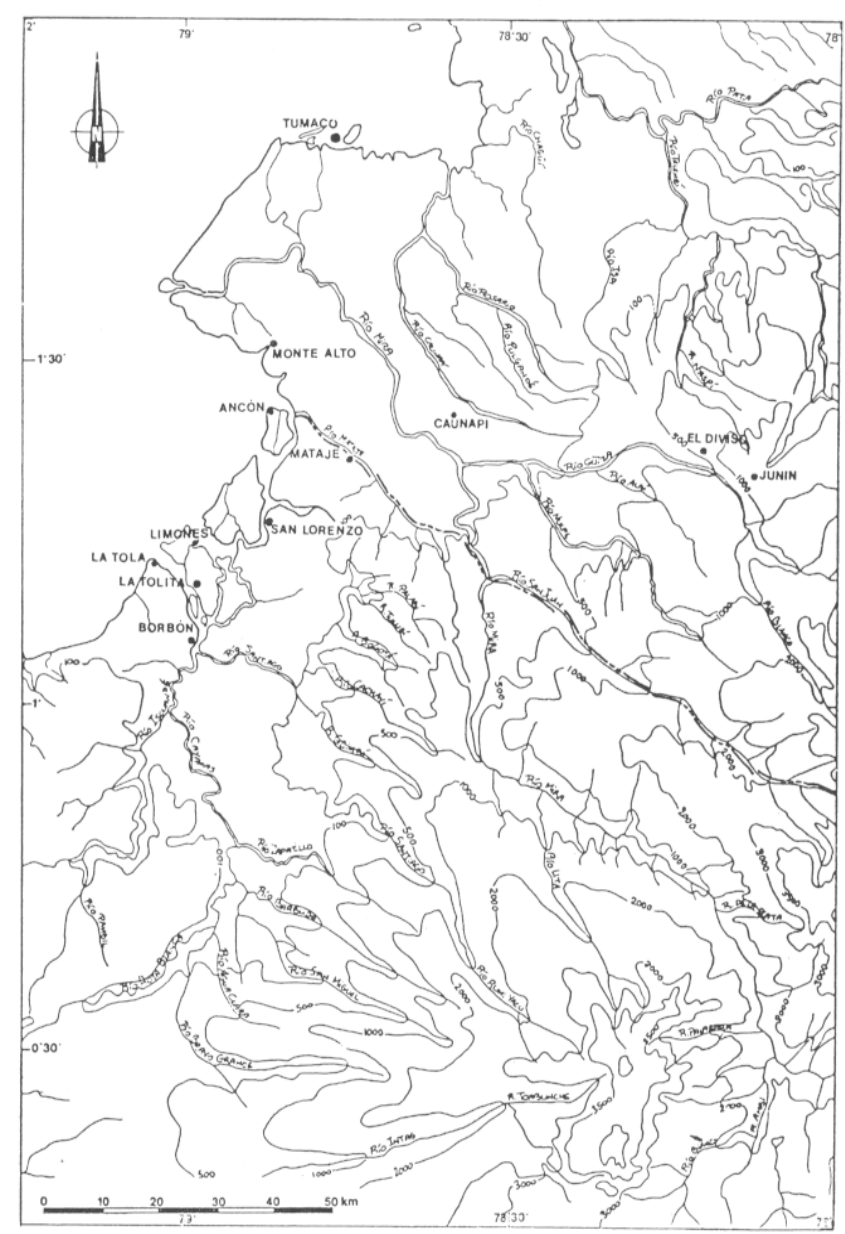

Fig. 1 - Situation des sites mentionnés. 
Dans ces projets, les objectifs recherchés ont été :

a) Obtenir une séquence de chronologie culturelle intégrable aux données chronologiques obtenues pour les régions voisines.

b) Établir à partir des corpus céramiques découverts en fouille une classification « ouverte » (susceptible d'être complétée par d'éventuelles fouilles ultérieures dans les régions proches).

c) Proposer une interprétation de l'évolution culturelle, des modes d'établissement et des moyens de subsistance caractérisant les divers groupes de ce littoral pacifique nord-équatorial.

d) Dans les deux projets, un aspect de formation aux recherches de terrain et de laboratoire pour des étudiants locaux a aussi été établi en coopération avec les organismes des deux pays.

Diverses études spécialisées, réalisées dans le cadre de ces projets, ont permis de préciser les résultats d'analyse empirique des vestiges archéologiques.

I - ÉTUDE DU MATÉRIEL ARCHÉOLOGI$Q U E$

\section{A - Classification de la céramique}

La classification des corpus céramiques du site Cancha H. 9 a suivi les principes de celle du projet Tumaco, publié en 1983.

La classification a porté sur le corpus Pré Tolita et sur le corpus Tolita. Le $1^{\text {cr }}$ corpus, classé en 29 types, a pu être rattaché à une tradition céramique du Formatif final. 19 de ces types se retrouvent dans le corpus Tolita et peuvent donc être considérés comme un tronc commun, mettant ainsi en évidence une filiation entre les deux périodes représentées par ces niveaux. Grâce au traitement informatisé effectué par G. Clément * au cours de l'année 1986, il a été possible de contrôler ces observations et de les comparer avec celles de la classification des corpus céramiques du programme Tumaco. Cette «étude spécialisée » a donc permis de démontrer par le calcul l'exactitude des observations qui soulignaient les relations entre les corpus des différents niveaux découverts dans la région de Tumaco et à La Tolita. Ces études par calcul informatisé seront publiées dans les synthèses en préparation.

Les études de composition par diffraction de rayons $X$, effectuées par J. Galvan, du C.S.I.C., Madrid (Conseil Supérieur de la Recherche Scientifi- que, Laboratoire d'Édafologie et Biologie végétale), ont aussi confirmé la validité de cette classification (Galvan, Martin, Galvan, Bouchard, 1989).

L'étude des figurines en céramique des deux niveaux du site Cancha a aussi corroboré les distinctions chrono-culturelles effectuées par classification des récipients en céramique.

Les figurines Pré Tolita représentent un style assimilable aux caractéristiques du Formatif final. Divers ornements, des coiffures, de même que le traitement de certaines parties des figurines constituent des traits diagnostiques de cette période. Les figurines du niveau Tolita, par contre, sont rattachables à la période culturelle suivante (phase «Tolita $»$, bien que leur facture soit relativement commune. Cette qualité assez médiocre est due à une fabrication au moule et en série.

\section{$B$ - Étude des vestiges d'orfèvrerie}

Les fragments d'orfèvrerie du niveau "Tolita » ont été analysés par D. Scott, chargé de recherche et d'enseignement à l'Institut d'Archéologie de l'Université de Londres (Department of archaeological conservation and material sciences).

Cette étude poursuit la coopération commencée lors du projet Tumaco pour l'analyse des composants métalliques de l'orfèvrerie de la côte Pacifique nordéquatoriale. Elles ont confirmé, pour le niveau Tolita, l'existence d'une orfèvrerie déjà développée, incluant la fabrication délibérée d'alliages divers à base d'or et de cuivre et d'incorporation de platine dans l'or en fusion (D. Scott, J.-F. Bouchard, 1989).

II - ÉTUDE DU MILIEU NATUREL : PALYNOLOGIE ET GÉOMORPHOLOGIE

En plus des études « archéologiques », le projet La Tolita a permis d'entreprendre, dans le cadre de la coopération franco-équatorienne, deux études du milieu naturel concernant la flore (palynologie des mangroves), et la dynamique du littoral autour de l'île de La Tolita. Elles constituent des " premières » pour ce milieu de mangrove, du Pacifique nordéquatorial.

Ces deux études ont permis de confirmer une stabilité globale du milieu naturel pendant la période d'occupation précolombienne ainsi que la formation relativement récente du complexe fluvio-deltaïque de 
l'embouchure du Santiago, où se trouve l'île de La Tolita. Ces données coïncident avec les schémas d'interprétation de l'évolution culturelle proposées par l'archéologie.

Ces missions ont été effectuées par :

- Étude palynologique de la région de La Tolita.

Claude Caratini, Colette Brisset, chercheurs C.E.G.E.T., C.N.R.S.

- Étude de la dynamique du littoral de la région de La Tolita.

Jean-Pierre Tihay, Maître de Conférences, I.R.S.A.M., Université de Pau et des Pays de l'Adour.

Les deux rapports seront intégrés comme « études spécialisées » aux publications de synthèse en préparation.

\section{III - RÉSULTATS OBTENUS: INTERPRÉTA-} TIONS

L'ensemble de ces recherches et études spécialisées permet de proposer une interprétation des résultats obtenus. Celle-ci peut se résumer de la façon suivante :

\section{Cadre de chronologie culturelle}

C'est vers 600-500 avant J.-C. qu'apparaissent des évidences d'une première occupation, reliée à la tradition "Chorrera». Ces premiers groupes seraient arrivés dans cette région à la suite de migrations originaires de la côte centre-sud de l'Équateur. Ils développèrent, sur les bases d'une tradition déjà presque déclinante, un faciès local, découvert dans le nord de la côte de la province d'Esmeraldas, en Équateur, et dans l'ensemble du département de Nariño, en Colombie.

Après quelques siècles (vers 300 av. J.-C. environ), ce faciès local donna naissance à une nouvelle tradition céramique, propre à la période suivante des Développements Régionaux. Cette tradition « Tolita » dura, environ, de 300 avant J.-C. à 300 après J.-C. avec une évolution, perceptible à travers les différents corpus. Après une première période de grande diffusion sur l'ensemble du littoral, vers le $2^{c}$ de notre ère elle ne subsiste plus qu'aux alentours de La Tolita. Ailleurs, apparaît alors une céramique différente, représentée par les corpus de
El Balsal, de Pampa de Nerete, puis de El Morro, en Colombie. Après 300 , on ne trouve plus de survivance de la «tradition La Tolita » sur cette côte.

Dans plusieurs sites de Colombie, ces niveaux culturels sont suivis par des occupations de la période d'Intégration. Elles sont datées vers 1000 après J.-C. et correspondent à la construction de monticules artificiels, les tolas, destinés à surélever les constructions. En Équateur, à La Tolita, le site Cancha n'a pas permis de découvrir d'occupations tardives. On ne peut donc pas encore proposer d'interprétation globale de l'archéologie de cette dernière période préhispanique pendant laquelle l'unité du littoral nord-équatorial paraît se défaire.

Les modèles d'établissement et les moyens de subsistance

Les caractéristiques du milieu naturel de la région nord-équatoriale ont influencé les modèles d'établissement pendant les différentes époques préhispaniques. Plusieurs sites ont permis de dégager des constantes dans ces modèles. Ils occupent, de préférence, une position de seuil à proximité de la mangrove, en bordure de la plaine alluviale. Ils sont presque toujours proches de cours d'eau et permettent l'accès direct aux réseaux de circulation et de pêche du milieu aquatique. La stratigraphie de plusieurs sites, fouillés au cours de différentes recherches, montre que les mêmes emplacements ont

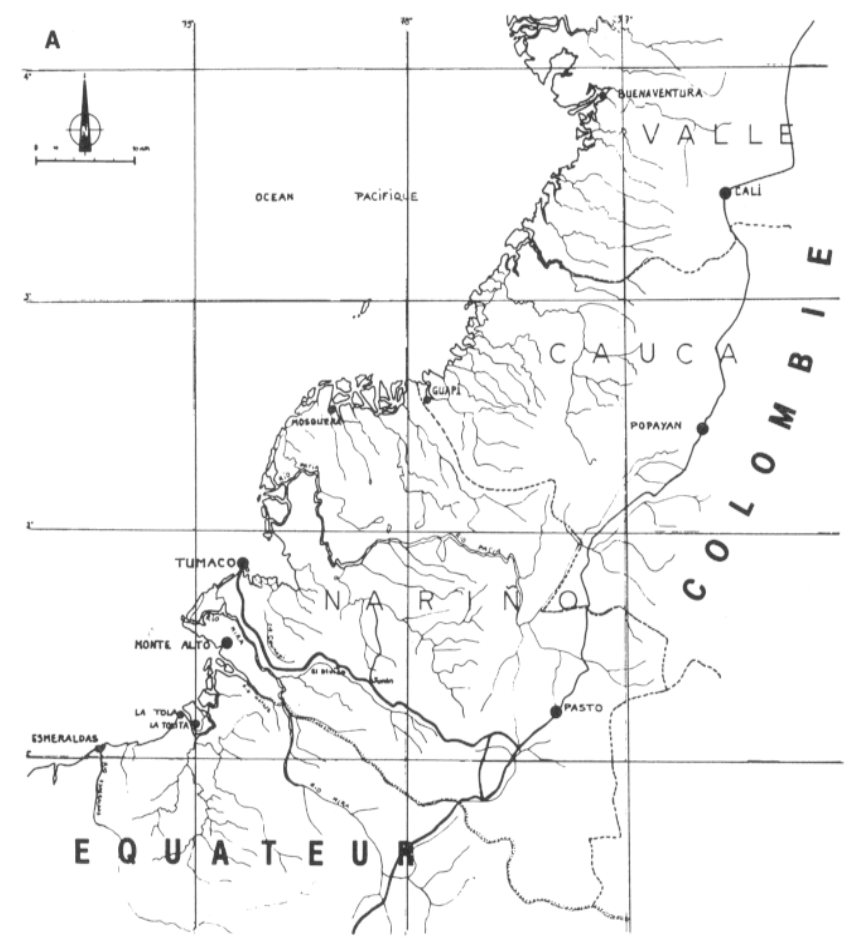

Fig. 2 - Détail de la région de Tumaco. 
souvent été réoccupés au cours du Formatif final et des Développements Régionaux.

Le milieu semi-aquatique de la frange littorale a aussi beaucoup influencé le choix des moyens de subsistance. Contrairement aux autres régions côtières, où on observe des options agricoles, «terrestres ", dès le passage à l'économie de production, l'exploitation des ressources des divers systèmes et milieux aquatiques (mer, chenaux de marée et rivières), semble être la base de l'économie.

Les évidences indirectes de pêche, comme les poids de filet, sont nombreuses tant dans les niveaux de la période du Formatif final que dans ceux de celle des Développements Régionaux. Les évidences directes sont plus rares, du fait que les détritus d'alimentation étaient évacués dans les cours d'eau ou dans la mer plutôt que dans les alentours des sites. Il existe toutefois certains restes osseux de poissons, parfois travaillés, de même que des couches ou lentilles de sédiment contenant des coquilles de mollusques comestibles.

Les observations actuelles montrent la grande importance de la pêche en mer et dans le cours inférieur des fleuves ainsi que dans les canaux de marée. Cette pêche concerne les poissons de grande taille, mais surtout les poissons de taille moyenne, souvent grégaires, ainsi que les crustacés (crevettes de tous genres et crabes). Pendant les époques de récession naturelle de ces activités, la pêche ne cesse pas et ce sont les coquillages qui apportent une quantité importante de protéines dans l'alimentation.

En plus de ces activités de pêche, des activités relevant aussi de la prédation entrèrent dans les moyens de subsistance de ces populations. Le milieu de forêt apporte, en effet, une assez grande variété de végétaux sauvages comestibles. Ce sont principalement des fruits tropicaux mais aussi des tiges et des pousses d'arbres et de palmiers. Il existe aussi, depuis les plus anciennes périodes, quelques évidences de production d'aliments. L'agriculture était connue dans la région côtière équatoriale depuis la moitié du $4^{c}$ millénaire avant J.-C. et il semble clair que les populations qui habitaient ce littoral nord-équatorial la pratiquaient. Divers instruments de mouture (meules et mains de meule) ont été découverts dans plusieurs sites de cette région. Ils indiquent, selon toute probabilité, la culture du maïs sur les meilleures terres. De même, nous pouvons supposer la culture de racines, comme le manioc.

Cette économie mixte constitue un exemple très particulier de l'économie des populations du littoral équatorial. Cette diversification, de même que la prédominance de la prédation sur la production agricole, en font une économie très originale par rapport à ce que nous savons de l'économie des autres groupes préhispaniques occupant le reste du littoral équatorial.

$$
\begin{array}{r}
\text { Chercheurs associés au projet : } \\
- \text { Archéologie }: \text { J.-F. BouCHARD } \\
\text { Missions de fouilles et de laboratoire } \\
\text { (Chargé de recherches au C.N.R.S.) } \\
\text { - Traitement informatisé : G. CLÉMENT } \\
\text { (ER 313, C.N.R.S., Paris) } \\
\text { - Détermination des composants minéralogiques } \\
\text { de la céramique : J. GALVAN } \\
\text { (C.S.I.C., Madrid) } \\
\text { - Orfèvrerie : D. SCOTT } \\
\text { (Institute of Archaeology, University of London) } \\
\text { - Palynologie : C. BRISSET et C. CARATINI } \\
\text { (C.E.G.E.T.-C.N.R.S., Bordeaux) } \\
\text { - Géomorphologie : J.-P. TiHAY } \\
\text { (Université de Pau et des pays de l'Adour) }
\end{array}
$$

Bouchard (1984) - Archéologie de la région de Tumaco, Colombie. Édition Recherches sur les Civilisations, $201 \mathrm{p}$. Travaux de l'Institut Français d'Études Andines, Mémoire $\mathrm{N}^{\circ} 34$. A.D.P.F. Paris.

BOUCHARD (1986) - Las más antiguas culturas precolombinas del Pacífico ecuatorial septentrional. Simposio del $45^{\circ}$ Congreso Internacional de Amcricanistas : Arqueología y etnohistoria del sur de Colombia y norte del Ecuador. Miscelanea Anthropológica Ecuatoriana, N ${ }^{\circ}$ 6, pp. 109-129.

BOUCHARD (1989) - Evidencias de relaciones interculturales en la región norte, (Sur de Colombia y Norte de Ecuador).

Galvan, Martin, Galvan, Bouchard (1989) - Estudio mineralogico de las ceramicas procedentes del yacimiento arqueologico de la Tolita. In : Relaciones interculturales en el área ecuatorial del Pacífico durante la época precolombina. $46^{\circ}$ Congreso Internacional de Americanistas. B.A.R. International Scries 503, Oxford.

Bouchard (1990) - Deux sites côtiers : Tumaco et La Tolita (Colombic-Équateur). Dossiers de l'Archéologie, No 145 , pp. 64-69.

SCOTT et BOUCHARD (1988) - Orfebrería prehispánica de las llanuras del Pacífico de Ecuador y Colombia. Boletín del Museo del Oro, Vol. 22, pp. 3-16. Bogotà. 